Canadıan Journal of

HealthTechnologies

December 2021 Volume 1 Issue 12

\title{
Viewpoint: Thank You to Our Readers and Contributors
}

\author{
Nicole Mittmann, Editor-in-Chief
}

This issue is an important milestone for the Canadian Journal of Health Technologies. It marks the final issue in volume 1 of our new journal. Before our inaugural year draws to a close, l'd like to thank all of our contributors, readers, and subscribers for your engagement over the past 12 months.

The team at CADTH outlined several ambitious objectives when we established the journal in January 2021. First and foremost, we aimed to standardize CADTH's scientific reports and make them more accessible through channels like PubMed and Google Scholar. As a major Canadian publisher of evidence and recommendations that influence pan-Canadian decisions about pharmaceuticals and health technologies, we are strongly committed to knowledge accessibility and mobilization.

Looking back through the 12 issues in volume 1, I'm proud to see the impressive range of CADTH evaluations covering topics that are all highly relevant to Canadian health systems:

- a watch list of emerging health technologies and trends

- a comprehensive report on the supply, distribution, technical operations, and clinical and research use of advanced medical imaging equipment in Canada

- a suite of health technology reviews relating to the diagnosis, treatment, and prevention of tuberculosis (which informed a CADTH condition-level review on tuberculosis)

- comprehensive health technology assessments (HTAs) and rapid reviews on a range of drugs, devices, and clinical interventions.

For the first time, we also published accepted abstracts from the annual CADTH Symposium. Our symposium is a signature meeting for the Canadian and global HTA communities.

Publishing the abstracts in a special supplement will make the proceedings more available to the scientific community and other stakeholders.

An important factor in our decision to establish a CADTH journal was the recognition of the necessity for a Canadian publication that could disseminate high-quality work from panCanadian producers and users of HTA to a wide audience. Therefore, another key objective for the Canadian Journal of Health Technologies is to foster collaboration and publication opportunities for Canadian experts, jurisdictions, and stakeholders in HTA. 
We could not have predicted the positive response to the journal or how quickly our colleagues would approach us with new opportunities. In May, the journal published Using Health Care Resources Wisely After the COVID-19 Pandemic, a joint report with Choosing Wisely Canada that explores consensus on evidence-based recommendations to reduce low-value care when health systems resume the services that were put on hold due to the COVID-19 pandemic. And, in November, we published Canadian Trends and Projections in Prescription Drug Purchases: 2001-2023, a peer-reviewed analysis by a group of national experts that provides important new insight on Canada's drug spending in the hospital sector and the public and private retail sectors.

Launching a new journal is no small feat, and we are indebted to our internal publishing team and staff from across CADTH for their enthusiasm for this journey and their commitment to the highest publication standards. The success the journal has achieved in 12 short months is a testament to their dedication.

Finally, I would like to encourage our readers to send us your comments and ideas for future issues of the Canadian Journal of Health Technologies. We believe the journal will be an effective communication channel for HTA, implementation science, and knowledge mobilization in Canada. We welcome your perspectives on how we can continue to advance this objective. 